\title{
El análisis cualitativo de nexos causales: El aporte del process-tracing a las ciencias de la gestión
}

\author{
Mario Marcello Pasco Dalla Porta', Vania Estefany Cárdenas \\ Vuckovic $^{2}$ y Ángello Augusto Hernández Aburto ${ }^{3}$
}

\author{
${ }^{1}$ Profesor Asociado del Departamento Académico de Ciencias de la Gestión, Pontificia \\ Universidad Católica del Perú, Perú | mpascod@pucp.pe | https://orcid.org/0000-0002-0799- \\ 1033 \\ ${ }^{2}$ Egresada de la Facultad de Gestión y Alta Dirección, Pontificia Universidad Católica del \\ Perú, Perú | vania.cardenas@pucp.pe | https://orcid.org/0000-0000-0000-0000 \\ ${ }^{3}$ Egresado de la Facultad de Gestión y Alta Dirección, Pontificia Universidad Católica del \\ Perú, Perú | angello.hernandez@pucp.edu.pe | https://orcid.org/0000-0000-0000-0000
}

\begin{abstract}
Resumen: La observación de nexos causales en los procesos organizacionales ha priorizado los enfoques cuantitativos a partir del procesamiento de bases de datos. El process-tracing (rastreo de procesos) es un diseño metodológico alternativo, predominantemente cualitativo, para el análisis de esos vínculos causales. Desafortunadamente, se trata de un método poco conocido y con limitada aplicación a la investigación de la realidad organizacional, más aún en la región iberoamericana. El propósito de este trabajo es examinar la relevancia de este método para el análisis de las organizaciones. Para ello, primero se abordan los fundamentos epistemológicos y metodológicos que sustentan este método. Seguidamente se examina su relevancia para el análisis de la gestión organizacional. Finalmente, se describe su aplicación para el estudio del proceso estratégico de una cadena de restaurantes con orientación vegetariana en Lima, Perú. El documento concluye resaltando la importancia de usar este método y su contribución al pluralismo metodológico en la disciplina.
\end{abstract}

Palabras clave: Process-tracing; Métodos de Investigación; Análisis Organizacional; Proceso Estratégico.

Qualitative Analysis of Causal Links: Contribution of Process-Tracing to the Management Sciences

\begin{abstract}
The observation of causal links in organizational processes has prioritized the quantitative approach based on the processing of datasets. Process-tracing is an alternative methodological design, predominantly qualitative, for analysing these causal bonds. Unfortunately, this method is barely known and has limited application in the investigation of the organizational reality in the lberoamerican region. The purpose of this paper is to examine the relevance of this method to the analysis of organizations. It departs by addressing the epistemological and methodological foundations of this method. Then it examines its importance to the analysis of organizations' management. Lastly, it describes its application to the study of the strategic process of a restaurant chain with a plant-based diet in Lima, Peru. The document concludes by highlighting the importance of using this method and its contribution to the methodological pluralism in the discipline.
\end{abstract}

Keywords: Process-tracing; Research Methods; Organizational Analysis; Strategic Process.

\section{Introducción}

La inferencia científica en las ciencias sociales ha sido ampliamente debatida. King, Keohane, \& Verba, (1994) distinguen las inferencias descriptivas (diferenciar patrones sistemáticos y no sistemáticos en los fenómenos analizados) de las causales (medir los efectos sistemáticos de un fenómeno sobre otro), y señalan que generar éstas es el propósito último de la investigación social (usualmente mediante análisis cuantitativo con muestras amplias). Brady \& Collier, (2010) cuestionan esta perspectiva y resaltan la importancia de hacer caracterizaciones a profundidad de los fenómenos estudiados. En esa línea, en las décadas recientes han surgido alternativas para el estudio de los nexos causales con un enfoque cualitativo. El process-tracing es una de ellas. 
El propósito de este trabajo es examinar la relevancia de este método para el análisis de la gestión organizacional. En esa línea, el artículo está dividido en tres partes: la primera aborda los fundamentos epistemológicos y metodológicos del process-tracing, la segunda examina su relevancia para el análisis organizacional, y la tercera describe su aplicación en un estudio de caso.

\section{Fundamentos Epistemológicos y Metodológicos}

Varios autores reportan un uso creciente de métodos basados en estudio de casos respecto de investigaciones con tamaños muestrales amplios (Gerring, 2006; George \& Bennet, 2005).

Gerring (2004) señala que un estudio de caso requiere "an in-depth study of a single unit (a relatively bounded phenomenon) where the scholar's aim is to elucidate features of a larger class of similar phenomena" (341). Yin (2008) añade que se trata de "an empirical inquiry that investigates a contemporary phenomenon in depth and within its real-life context" (s.n.). Son rasgos distintivos de este método un tamaño muestral reducido, recolección intensiva de información, diferentes fuentes de información, énfasis en nexos causales e importancia del contexto (Yin, 2008; Gerring, 2004; Stake, 1994).

Pierson (2003) argumenta que los procesos sociales ocurren con temporalidades heterogéneas (ver Tabla 1), por lo cual la mayor parte de la investigación social se enfoca en procesos de corto plazo (cuadrante I). Esto motiva su llamado a estudiar procesos que se desenvuelven de forma más lenta, examinando sus causas y efectos acumulativos, cadenas causales y umbrales.

Tabla 1. Horizontes de tiempo en diferentes aproximaciones causales.

\begin{tabular}{cccc}
\hline & & Horizonte de tiempo del resultado \\
\hline & & Corto & Largo \\
Corto & I & Efectos \\
$\begin{array}{c}\text { Horizonte de } \\
\text { tiempo de la } \\
\text { causa }\end{array}$ & & Procesos de & acumulativos \\
& Largo & Corto plazo & IV \\
& & Umbrales y & Causas \\
\hline
\end{tabular}

Fuente: Adaptado de Pierson (2003)

El process-tracing busca examinar nexos causales en procesos sociales complejos a lo largo del tiempo, usualmente dentro de un estudio de caso. Su lógica epistémica es "to identify the intervening causal process - the causal chain and causal mechanismbetween an independent variable (or variables) and the outcome of the dependent variable" (Bennet, 2005: 206). Bennet (2010) destaca 'the examination of 'diagnostic' pieces of evidence within a case that contribute to supporting or overturning alternative explanatory hypotheses" (208).

La solidez metodológica del process-tracing se sostiene en la eliminación progresiva de las causas potenciales, la apertura a la equifinalidad, la identificación de trayectorias causales, la reducción de los errores inferenciales (en particular aquellos vinculados a variables espurias) y la elucidación de casos divergentes (George \& Bennet, 2005).

Este método se caracteriza por el abordaje complejo similar al detectivesco, el énfasis en nexos causales, la combinación de diferentes fuentes de evidencia para testear las inferencias, el esclarecimiento de cadenas causales, la incomparabilidad de las piezas de evidencia, y la indeterminación sobre el número total de observaciones (Gerring, 2006). 
Un aspecto fundamental es el análisis minucioso de los vínculos causales. Con ese propósito, Van Evera (1997) ha diseñado una herramienta útil, de corte bayesiano, para probar las evidencias empíricas en función de su nivel de necesidad/suficiencia. Esto conduce a las cuatro pruebas de causación mostradas en la Tabla 2. La prueba straw in the wind solo garantiza una evidencia débil respecto del vínculo causal estudiado, las pruebas hoop y smoking gun ofrecen evidencias más consistentes al respecto, y la prueba doubly decisive proporciona evidencias de gran solidez. Desafortunadamente, Bennet (2010) señala que las evidencias para pasar esta última prueba son infrecuentes en la investigación social, por lo que una combinación de pruebas hoop y smoking gun es la mejor alternativa para generar inferencias válidas.

Tabla 2. Cuatro pruebas de causación.

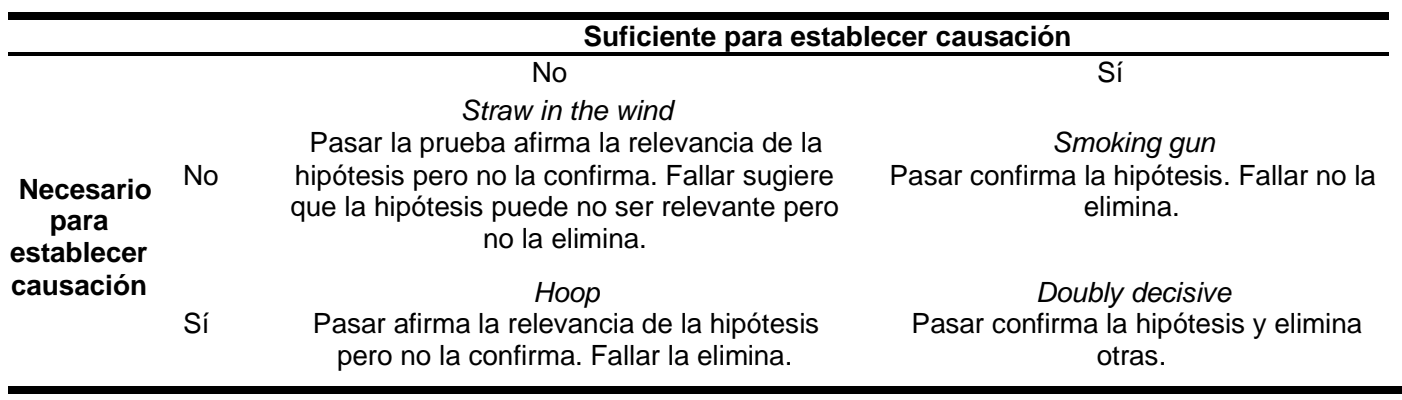

Fuente: Adaptado de Bennet (2010) y Van Evera (1997)

Este método enfrenta algunas limitaciones. Como usualmente funciona anidado en un estudio de caso, su poder explicativo se circunscribe al contexto analizado, con limitada generalización (Bennet, 2010). La posibilidad de desagregar indefinidamente los mecanismos causales también requiere cierta atención (King, Keohane, \& Verba, 1994). Además, la dependencia en los procesos de codificación de la data cualitativa requiere particular cuidado sobre cuestiones de confiabilidad y sesgos interpretativos.

\section{Relevancia para el Análisis Organizacional}

A pesar de que el process-tracing es un método ampliamente utilizado en otras ciencias sociales desde hace varias décadas, su uso en las ciencias de la gestión ha sido bastante limitado. En los últimos 25 años solo se han producido 107 publicaciones indizadas en Scopus y 71 en Web of Science, vinculadas a temas de gestión y negocios, en los que el process-tracing aparece mencionado en los descriptivores básicos (título, resumen y palabras clave) (ver Figura 1).

En estos estudios, la mayoría tiene un enfoque cuantitativo (testeo experimental mediante simulación por computadora, rastreo del cursor, registro de navegación, rastreo ocular y protocolos verbalizados, entre otros), y solo 13 publicaciones en Scopus y 11 en Web of Science adoptan la aproximación cualitativa materia del presente trabajo, todas ellas publicadas recién en los últimos cinco años. 


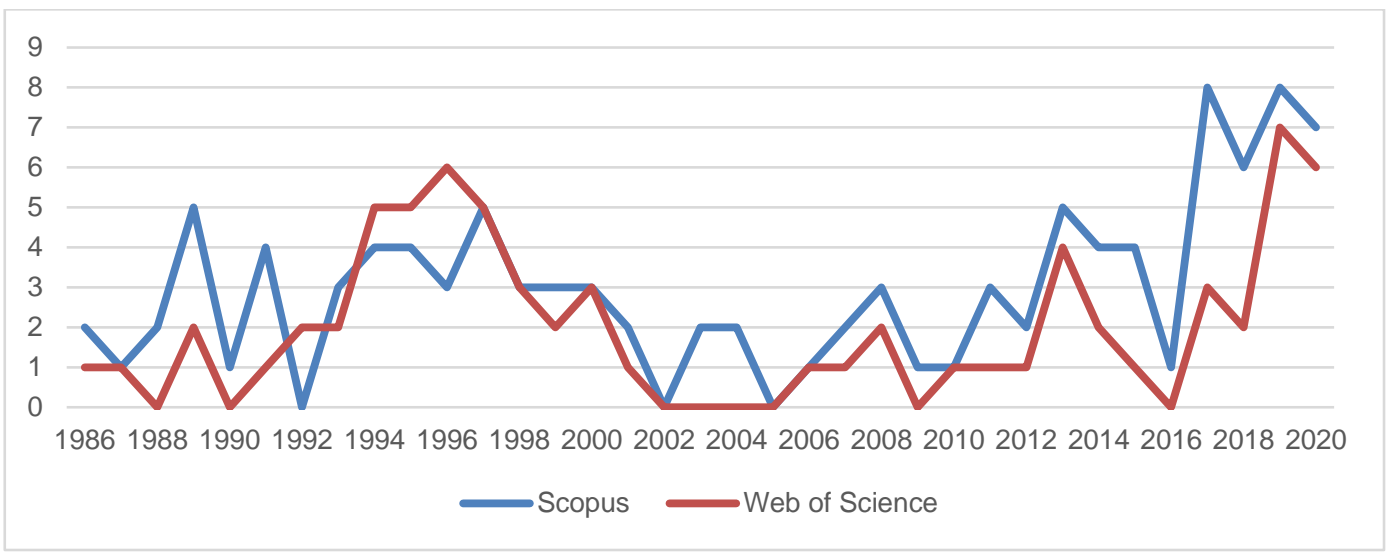

Fig. 1. Artículos que involucran el process-tracing vinculados a temas de gestión y negocios.

Estos estudios cubren un amplio espectro de temas que incluyen innovación (Liu, 2019; Di Paola \& Spena, 2019; Makarevich, 2017), sostenibilidad (Muñoz, Cacciotti, \& Cohen, 2018; Dossa \& Kaufer, 2014), diseño de procesos (Soutrisna \& Goulding, 2019), planeamiento (Derbyshire, 2020), capacidad institucional (Evans, 2017), cambio organizacional (Kusano \& Sanada, 2019), gestión de riesgo (Timmer \& Kaufmann, 2019), alianzas estratégicas (Rey-García, Calvo, \& Mato-Santiso, 2019), gestión de stakeholders (Nguyen, Chileshe, Rameezdeen, \& Wood, 2019) y satisfacción del consumidor (Tessem, 2018). Conviene notar que todos estos trabajos están publicados en inglés y no se ocupan de organizaciones en la región iberoamericana.

Aunque ninguno de estos trabajos se enfoca en el método como tal, varios de ellos aportan elementos a considerar sobre su relevancia disciplinaria. En términos generales, pueden identificarse varias razones por las cuales el process-tracing es valioso para el estudio de la gestión organizacional: a) la creciente complejidad de las organizaciones y del entorno en el que se desempeñan, lo cual requiere abordajes metodológicos que analicen la complejidad de la empresa, su entorno y cómo esto influye en la toma de decisiones; b) la relevancia del análisis causal en la disciplina, considerando el rol decisivo que tienen la toma de decisiones y la generación de resultados; c) la naturaleza multicausal de la gestión organizacional, la cual requiere la identificación y contraste de explicaciones alternativas y diversos tipos de evidencias; y d) la importancia de la agencia en las organizaciones, teniendo en cuenta el creciente interés por la diversidad y roles de los distintos stakeholders.

En cuanto a la forma de implementación del process-tracing, las pautas más recurrentes de diseño y aplicación de este método incluyen: a) la aproximación abductiva para el refinamiento de postulados teóricos (Kusano \& Sanada, 2019; Nguyen, Chileshe, Rameezdeen, \& Wood, 2019); b) el uso de un número limitado de casos (Soutrisna \& Goulding, 2019); c) la combinación de distintas técnicas de recolección de información, incluyendo entrevistas a profundidad, grupos focales, observaciones, revisión de archivos y reportes en medios, entre otros (Liu, 2019; Tesssem, 2018; Dossa \& Haeufer, 2014); d) la obtención de datos sobre periodos largos de tiempo y la identificación de trayectorias temporales (Soutrisna \& Goulding, 2019; Liu, 2019; Tessem, 2018); e) la aplicación de pruebas de causación como las planteadas por Van Evera (Derbyshire, 2020; Tessem, 2018), con particular atención a la equifinalidad (Kusano \& Sanada, 2019); y f) el uso de narraciones pormenorizadas y descripciones densas de los procesos investigados (Kusano \& Sanada, 2019; Soutrisna \& Goulding, 2019; Evans, 2017). 
Finalmente, es importante añadir que este método también está ausente en la literatura sobre métodos de investigación en gestión, como puede constatarse tras revisar los principales textos metodológicos en la disciplina (Cassell \& Cunliffe, 2018; Gupta \& Awasthy, 2015; Weathington, Cunningham, \& Pittenger, 2012; Saunders, Lewis, \& Thornhill, 2009; Broussine, 2008; Greener, 2008; Adams, Khan, Raeside, \& White, 2007; Neergaard \& Parm, 2007; Tharenou, Donohue, \& Cooper, 2007; Quinton \& Smallbone, 2006; Lancaster, 2005; Thomas, 2003; Partington, 2002; Thietart \& Wauchope, 2001; Gummesson, 1999).

\section{Aplicación a un Estudio de Caso}

\subsection{Diseño Metodológico}

La gastronomía peruana ha experimentado un boom en la última década, reflejado en la notable ampliación de la oferta gastronómica en cuanto a la diversidad de ingredientes, preparación de los platos, servicio al cliente, infraestructura de los locales y desarrollo de prácticas sostenibles (Matta \& García, 2019). Sin embargo, el sector enfrenta retos importantes. Varios expertos coinciden en que muchos restaurantes carecen de una perspectiva estratégica que oriente su gestión y presentan una alta tasa de mortalidad (Kisic, 2015; León de Peralta, 2015; Medina, 2015). Desafortunadamente, es escasa la literatura que examine en detalle el proceso estratégico en este tipo de empresas y su conexión con el desempeño organizacional.

El estudio de caso se centró en el análisis del proceso estratégico de Veggie Pizza, una cadena de pizzerías con orientación vegetariana (plant-based diet). ${ }^{1}$ Esta empresa es interesante por su propuesta de valor distintiva (es la única cadena con esa orientación), su rápida expansión y el abanico de decisiones estratégicas adoptadas a lo largo del tiempo con distintos niveles de efectividad.

El propósito del rastreo de procesos fue indagar los efectos de las principales decisiones estratégicas adoptadas y los mecanismos causales involucrados en el desempeño de la cadena de restaurantes.

A pesar de que el análisis del proceso estratégico en las pequeñas empresas permite una mejor dirección de la organización, fomenta su competitividad y contribuye a su sostenibilidad, ha sido escasamente abordado (Ijir y Gbegi 2015; Wang et al., 2007; Sandberg et at., 2001). El marco analítico para este estudio tomó en cuenta diversos enfoques teóricos sobre el proceso estratégico en las organizaciones (David, 2013; Kaplan y Norton, 2008; Mcmillan y Tampoe, 2001), con énfasis en las herramientas estratégicas correspondientes a las etapas de planeamiento (visión, misión, valores, análisis del micro y macro entorno, y análisis de fortalezas, oportunidades, debilidades y amenazas), implementación (objetivos de corto plazo, asignación de recursos y estructura organizacional) y evaluación (balanced scorecard).

Un aspecto importante del process-tracing es la recolección intencional de múltiples piezas de evidencia. En ese sentido, se realizaron once entrevistas en profundidad con actores clave de la organización (dos socios fundadores, dos gerentes de local, un encargado de cocina y seis trabajadores), se hicieron dos entrevistas adicionales con expertos del sector (de otras cadenas de restaurantes), se consultaron los archivos de la empresa (manuales internos, portal web y redes sociales), y se sistematizó información externa (reportes periodísticos, notas en revistas gastronómicas y críticas culinarias).

${ }^{1}$ Este estudio fue desarrollado como parte de una investigación de tesis de licenciatura desarrollada por Cárdenas Vuckovic \& Hernández Aburto (2019) para el programa académico de gestión en la Pontificia Universidad Católica del Perú. 


\subsection{Análisis de Resultados}

Toda la información cualitativa recolectada fue codificada mediante el software Atlas.ti. Las categorías teóricas extraídas de la literatura fueron ampliadas con las categorías emergentes identificadas en el caso. Se prestó particular atención a las decisiones estratégicas adoptadas (apertura de locales, alianzas estratégicas, diseño de operaciones, gestión del personal y estrategias de marketing), los resultados alcanzados (expansión, posicionamiento y cierre de locales) y los mecanismos causales involucrados (tácticas especificas correspondientes a cada decisión estratégica). El equipo de investigación estuvo conformado por dos tesistas, lo cual permitió hacer una doble codificación a fin de reducir posibles sesgos en la interpretación de la información. Todo el proceso de codificación y análisis fue supervisado por el asesor de la tesis.

El primer paso del análisis consistió en establecer la línea de tiempo de los procesos investigados. De ese modo, se identificaron las principales decisiones estratégicas durante el periodo 2014-2017, diferenciadas por las áreas de gestión correspondientes (ver Figura 2).

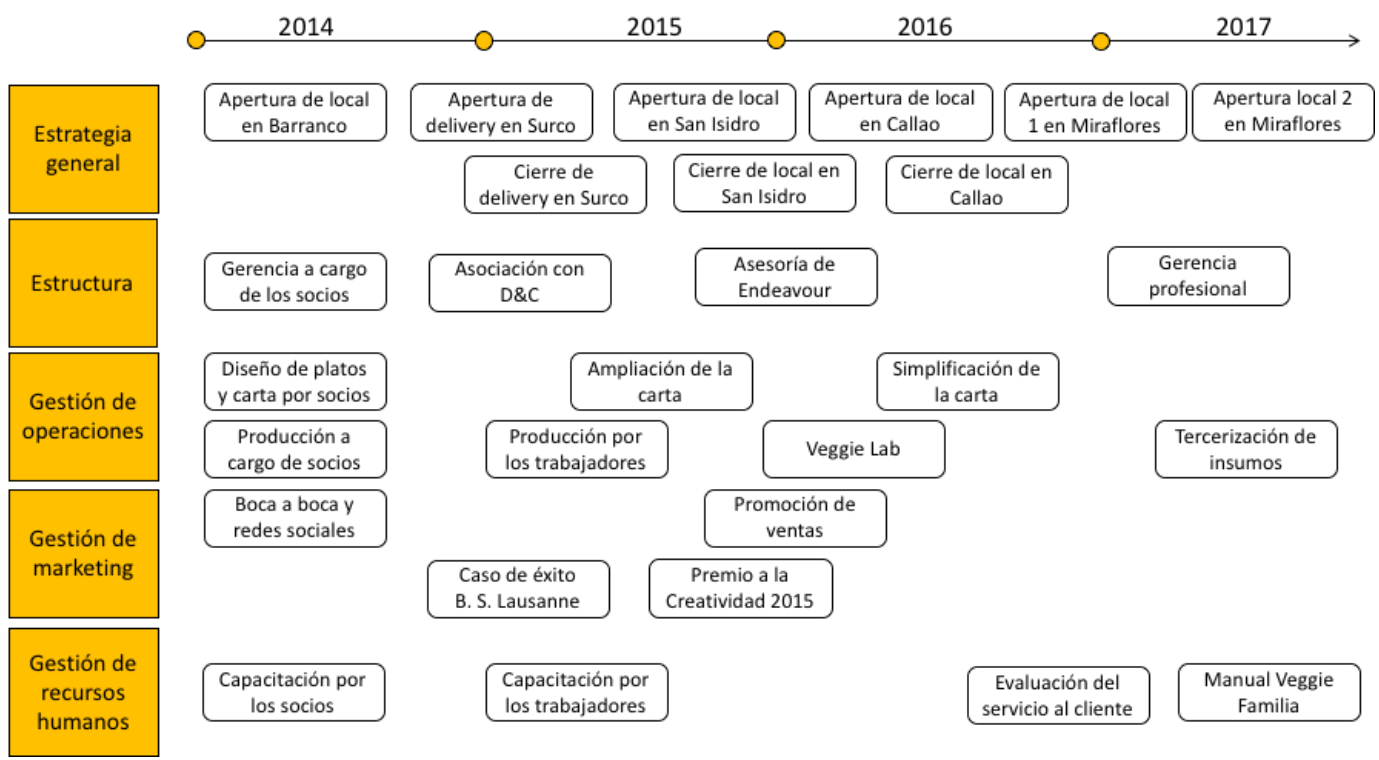

Fig. 2. Línea de tiempo de las decisiones estratégicas de Veggie Pizza (2014-2017) Adaptado de Cárdenas \& Hernández (2019)

Seguidamente se diferenciaron los procesos analizados. En esa línea, las distintas decisiones estratégicas adoptadas en la empresa fueron agrupadas analíticamente en tres fases. La fase de decisiones intuitivas de apertura comprendió el diseño de la carta, la apertura del primer local, la conducción directa del negocio por los socios fundadores y el uso apropiado de las redes sociales, lo cual posibilitó el exitoso posicionamiento del negocio. La fase de decisiones intuitivas de crecimiento incluyó la rápida apertura de nuevos locales, la ampliación de la carta y la delegación de algunos procesos clave a los trabajadores, lo cual eventualmente afectó la propuesta de valor y condujo al cierre de estos locales. La fase de decisiones de reenfoque estratégico implicó el retorno a la estrategia inicial, la recepción de asesoría especializada, la contratación de un gerente con experiencia en el rubro, la apertura planificada de dos nuevos locales y la inclusión de mejoras en la gestión de operaciones, lo cual permitió un crecimiento sostenible de la empresa.

El paso siguiente consistió en aplicar las pruebas de causación. En cada fase se seleccionaron algunas decisiones clave y los resultados correspondientes. Seguidamente se aplicaron las cuatro pruebas de causación indicadas en la Tabla 2. En cada prueba se diferenciaron los mecanismos causales involucrados con base en las distintas evidencias identificadas. 
Finalmente, se ponderaron los hallazgos en función de la solidez relativa de las evidencias, priorizando las pruebas smoking gun y hoop, dado que no se encontraron evidencias para pasar la prueba más exigente. Estas pruebas

Las pruebas de causación aplicadas permitieron elucidar los nexos causales ${ }^{2}$. Los resultados de estas pruebas fueron interpretados y reportados mediante narraciones pormenorizadas. En la primera de las fases identificadas se analizó el efecto de las decisiones intuitivas de apertura sobre la generación de ganancias estables del negocio (ver Tabla 3). Se encontró que la identificación de un nicho de mercado en crecimiento, la ubicación estratégica del local en un distrito altamente comercial y con una importante afluencia turística, el diseño innovador del menú, la adecuada gestión de las redes sociales y las relaciones públicas (incluyendo la participación de la empresa en el primer festival de veganismo en el país) fueron cruciales para el éxito inicial del negocio. Todo esto permitió el rápido posicionamiento de la empresa y un incremento sustantivo en sus ventas, permitiéndole incluso ganar el premio a la creatividad empresarial en 2014.

Tabla 3. Pruebas de causación sobre el efecto de las decisiones intuitivas de apertura sobre la generación de ganancias estables

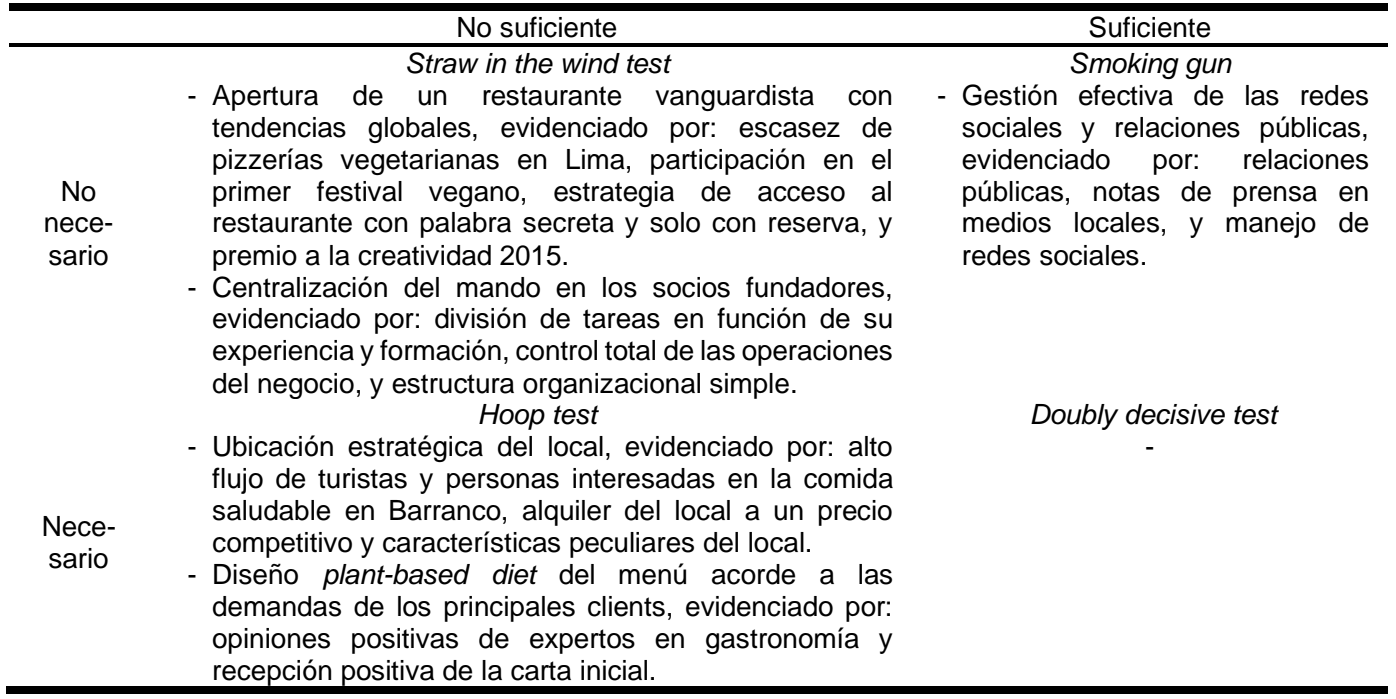

Adaptado de Cárdenas y Hernández (2019).

En la segunda fase se examinó el efecto de las decisiones intuitivas de crecimiento sobre el cierre de los locales (ver Tabla 4). Se constató que el apalancamiento financiero obtenido por la alianza con el grupo empresarial D\&C posibilitó el crecimiento de la organización, pero la apertura de nuevos locales fue inorgánica y se aplicaron parámetros distintos de los inicialmente establecidos. En esa línea, las críticas gastronómicas de ese año mostraron un descontento por apartarse del modelo inicial. Adicionalmente, la plana directiva experimentó cambios recurrentes pues se contrataron gerentes con experiencia en restaurantes de fast food, negocios con un formato distinto del planteado por Veggie Pizza. Este crecimiento inorgánico provocó variaciones en el diseño de las operaciones y en la forma de capacitación del personal, lo cual afectó negativamente la propuesta de valor y la imagen de la empresa, comprometiendo la sostenibilidad de varios de los nuevos locales.

2 El recuento presentado a continuación es una síntesis de los principales hallazgos encontrados. El detalle puede consultarse en Cárdenas y Hernández (2019). 
Tabla 4. Pruebas de causación sobre el efecto de las decisiones intuitivas de crecimiento sobre la clausura de locales

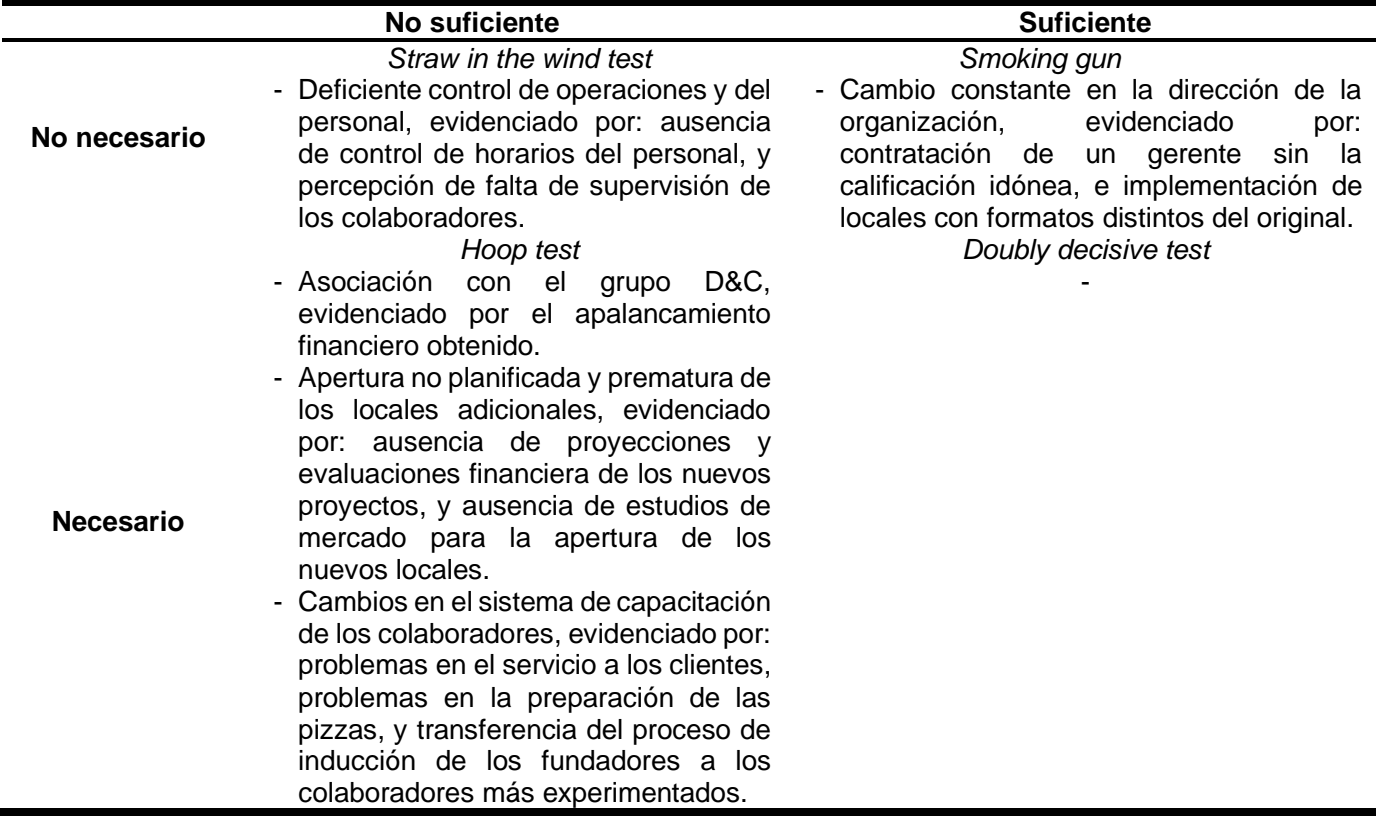

Adaptado de Cárdenas y Hernández (2019).

Finalmente, en la tercera fase se investigó el efecto de las decisiones de reenfoque estratégico sobre la gestión estable y efectiva de la organización (ver Tabla 5). Se evidenció que la mayor rigurosidad del proceso de selección del staff directivo, a cargo de profesionales idóneos, permitió una toma de decisiones con mayor orientación estratégica. Los principales cambios incluyeron el retorno a la estrategia inicial del negocio, para lo cual fue crucial una consultoría especializada facilitada por la aceleradora de proyectos Endeavor. Otros cambios importantes fueron la apertura planificada de dos nuevos locales comerciales, el manejo profesional de los insumos e inventarios mediante la creación de una nueva empresa encargada específicamente de su abastecimiento, y la implementación de manuales para uniformizar la capacitación a los trabajadores y el servicio al cliente. El resultado fue el reposicionamiento y crecimiento orgánico de Veggie Pizza.

Tabla 5. Pruebas de causación sobre el efecto de las decisiones intuitivas de crecimiento sobre la clausura de locales

\begin{tabular}{|c|c|c|}
\hline & No suficiente & Suficiente \\
\hline No necesario & $\begin{array}{l}\text { Straw in the wind test } \\
\text { - Mejoras en el control del personal, } \\
\text { evidenciado por cambios en el manejo } \\
\text { del personal, y presencia activa de } \\
\text { managers en los locales. } \\
\text { Hoop test } \\
\text { - Tercerización de la producción de } \\
\text { insumos, evidenciado por: apertura de } \\
\text { Veggie Lab y del Centro de Producción } \\
\text { Comma, estandarización de la } \\
\text { producción, mejoras en el control de } \\
\text { los inventarios, mejoras en la } \\
\text { negociación con los proveedores, y } \\
\text { reducción de las mermas. } \\
\text { - Cierre y apertura estratégica de } \\
\text { locales, evidenciado por: mentoría de } \\
\text { Endeavor, y decisión en función de } \\
\text { variables de competitividad: espacio y } \\
\text { ubicación. }\end{array}$ & $\begin{array}{l}\text { Smoking gun } \\
\text { - Mayor exigencia en la selección del } \\
\text { personal para la gerencia general, } \\
\text { evidenciado por: selección con base en la } \\
\text { capacidad de manejo logístico y financiero. } \\
\text { Doubly decisive test } \\
\text { - }\end{array}$ \\
\hline
\end{tabular}

Adaptado de Cárdenas y Hernández (2019). 
Finalmente, se examinaron las limitaciones metodológicas del estudio. Debido a que el análisis se centra en un caso, es importante precisar que los resultados se circunscriben al contexto investigado y tienen limitada generalización. Asimismo, aunque se usó un conjunto amplio de evidencias, se tuvo acceso limitado a documentos estratégicos y financieros, por lo que esta información tuvo que ser complementada mediante entrevistas. Algo similar ocurrió con el acceso a algunos actores clave (como exgerentes), lo cual hubiera ampliado el potencial explicativo de la investigación.

\section{Discusión}

Este trabajo presenta el process-tracing como una valiosa alternativa para examinar, con un enfoque cualitativo, los nexos causales en los procesos sociales (Bennet, 2005). A nivel epistemológico, este método permite la comprensión de cadenas de eventos y la elucidación de los mecanismos causales involucrados, a menudo desde un enfoque de complejidad y multicausalidad (George \& Bennet, 2005) que toma en cuenta la evolución paulatina de los procesos investigados (Pierson, 2003). A nivel metodológico, el rastreo de procesos se apoya en estudios de caso en profundidad, con múltiples fuentes de evidencia, para discriminar explicaciones alternativas de los fenómenos estudiados (Bennet, 2010; Van Evera, 1997).

El process-tracing viene siendo utilizado con creciente frecuencia en las ciencias de la gestión y el estudio organizacional, incluyendo varios trabajos sobre cuestiones estratégicas (Derbyshire, 2020; Kusano \& Sanada, 2019; Nguyen, Chileshe, Rameezdeen, \& Wood, 2019; Rey-García, Calvo, \& Mato-Santiso, 2019; Muñoz, Cacciotti, \& Cohen, 2018; Tessem, 2018; Dossa \& Kaufer, 2014). Desafortunadamente, son escasos los trabajos que se ocupan de analizar sus fortalezas metodológicas, ponderar su aporte a la disciplina, e ilustrar analíticamente su aplicación en estudios concretos, más aún en la región iboeroamericana. Este trabajo buscó reducir esa brecha mediante la caracterización de este método, sus herramientas y pautas de implementación en las fases de diseño metodológico y análisis de resultados.

En el caso investigado, el rastreo de procesos permitió explicar en detalle la forma en que las decisiones estratégicas adoptadas condujeron a los resultados reportados. El análisis reveló que las decisiones intuitivas e inorgánicas (expansión apresurada de la organización y limitada profesionalización de la gerencia) fueron condición suficiente para comprometer la sostenibilidad del negocio. En cambio, las decisiones basadas en un adecuado entendimiento del contexto (análisis de competitividad) y la planificación de la estrategia en áreas críticas (estandarización de operaciones y capacitación del equipo) fueron condición necesaria para generar mejores resultados (David, 2013; Macmillan \& Tampoe, 2001). Al posibilitar la identificación de relaciones causales específicas y diferenciadas en los procesos analizados, el process-tracing posibilita también que los gestores pueden incorporar ajustes más precisos en su accionar estratégico.

Sobre esa base, esta investigación espera hacer una contribución al pluralismo metodológico en las ciencias de la gestión así como al fomento de métodos que permitan una comprensión y explicación más amplia de la compleja realidad organizacional.

\section{Agradecimientos}

Los autores agradecen al Grupo de Epistemología de la Gestión y Teoría Organizacional de la Pontificia Universidad Católica del Perú por los comentarios y sugerencias sobre este estudio. Del mismo modo, se extiende el agradecimiento a todos participantes de la investigación y a los expertos consultados por la valiosa información proporcionada. 


\section{Referencias}

Adams, J., Khan, H.T.A., Raeside, R., \& White, D. (2007). Research Methods for Graduate Business and Social Science Students. Londres: SAGE Publications.

Bennet, G. (2010). "Process Tracing and Causal Inference”. En H. Brady, \& D. Collier. (Eds.), Rethinking Social Inquiry. Diverse Tools, Shared Standards. Segunda edición (pp. 207-219). Lanham, MD: Rowman \& Littlefield Publishers, Inc.

Brady, H. E., \& Collier, D. (Eds). (2010). Rethinking Social Inquiry. Diverse Tools, Shared Standards. Segunda edición. Lanham, MD: Rowman \& Littlefield Publishers, Inc.

Broussine, M. (2008). Creative Methods in Organizations Research. Londres: SAGE Publications.

Cárdenas Vuckovic, Vania Estefany, \& Hernández Aburto, Angello Juan Augusto. (2019). Análisis y evaluación del proceso estratégico de una cadena de restaurantes: caso de estudio Veggie Pizza. Tesis para optar por el título de licenciado en gestión. Lima: Pontificia Universidad Católica del Perú.

Cassell, C., Cunliffe, A.L., \& Grandy, G. (Eds). (2018). The SAGE Handbook of Qualitative Business and Management Research Methods. Londres: SAGE Publications.

David, F. R. (2013). Conceptos de Administración Estratégica. México: Pearson Educación Editorial.

Derbyshire, J. (2020). Cross Fertilising Scenario Planning and Business History by Process Tracing Historical Developments: Aiding Counterfactual Reasoning and Uncovering. Business History. DOI: https://doi.org/10.1080/00076791.2020.1844667

Di Paola, N., \& Russo Spena, T. (2019). What Drives Biopharmaceutical Firms' Exploratory Opennes? A Comparative Process Tracing Approach to the Analysis of R\&D Microfoundations, Journal of Business Research, 97, 94-103. DOI: https://doi.org/10.1016/j.jbusres.2018.12.004

Dossa, Z., \& Kaeufer, K. (2014). Understanding Sustainability Innovations Through Positive Ethical Networks. Journal of Business Ethics, 119, 543-559. DOI: https://doi.org/10.1007/s10551 013-1834-8

Evans, A. M. (2017). Building Institutional Capacity: From Pervasive Individualism to Sustained Coordination in Small Firm Sectors. Business and Politics, 15(2), 163-186, DOI: https://doi.org/10.1515/bap-2012-0041

George, A., \& Bennet., A. (2005). Process Tracing and Historical Explanation. En H. Brady, \& D. Collier. (Eds.), Rethinking Social Inquiry. Diverse Tools, Shared Standards. Segunda edición (pp. 205-232). Lanham, MD: Rowman \& Littlefield Publishers, Inc.

Gerring, J. (2004). What Is a Case Study and What Is It Good for? American Political Science Review, 98(2), 341-354.

Gerring, J. (2006). Case Study Research. Principles and Practices. New York: Cambridge University Press.

Greener, S. (2008). Business Research Methods. Brighton: Ventus Publishing.

Gummesson, E. (1999). Qualitative Methods in Management Research. Londres: SAGE Publications.

Gupta, R. K., \& Awasthy, R. (2015). Qualitative Research in Management. Methods and Experiences. Londres: SAGE Publications.

ljir, C. \& Gbegi, D. (2015). The role of strategic planning in small businesses in Benue State. International Journal of Public Administration and Management Research, 3(2), 10-20. Recuperado de: https://rcmss.com/2015/ijpamr/december/The\%20Role\%20of\%20Strategic\%20Planning\%20i n\%20Small\%20Business\%20Enterprises\%20in\%20Benue\%20State\%20Nigeria.pdf

Kaplan, R. S., \& Norton, D. P. (2008). The Execution Premium. Barcelona: Ediciones: Deusto. 
King, G., Keohane,R.O., \& Verba, S. (1994). Designing Social Inquiry. Scientific Inference in Qualitative Research. Princeton: Princeton University Press.

Kisic, Franco. (19 de enero de 2018). IK Restaurante: el concepto de una pasión. PQS \# La Voz del Emprendedor. Recuperado de: http://www.pqs.pe/historias/ik-restaurante-concepto-deuna-pasion

Kusano, M, \& Sanada, M. (2019). Crisis and Organizational Change: IASBs Response to the Financial Crisis. Journal of Accounting \& Organizational Change, 15(2), 278-301. DOI: https://doi.org/10.1108/JAOC-02-2018-0019

Lancaster, G. (2005). Research Methods in Management. A Concise Introuction to Research in Management and Business Consultancy. Oxford: Elsevier.

León de Peralta, D. (2015). El negocio de la gastronomía. ¿Qué tan sexy es de verdad la industria más sexy del Perú? Semana Económica. Lima: Perú Económico.

Liu, J. (2019). The Roles of Emerging Multinational Companies Technology-Driven FDls in their Learning Processes for Innovation. A Dynamic and Contextual Perspective. International Journal of Emerging Markets, 14(1), 91-114. DOI: https://doi.org/10.1108/IJoEM-07-20170232

Makarevich, A. (2017). Organizing for Success in Internal Corporate Venturing: An Inductive Case Study of a Multinational Consumer Goods Company, Creativity and Innovation Management, 26(2), 189-201. DOI: https://doi-org.ezproxybib.pucp.edu.pe/10.1111/caim.12213

Matta, R., \& García, M.E. (2019). The Gastro-Political Turn in Peru. Anthropology of Food, 14, 111. DOI: http://journals.openedition.org/aof/10061

Macmillan, H., \& Tampoe, M. (2001). Strategic Management: Process, content and implementation. Oxford: Oxford University Press.

Medina, I. (7 de agosto de 2015). La crítica de Ignacio Medina al Veggie Pizza. El Comercio. Recuperado de: https://elcomercio.pe/gastronomia/noticias/critica-ignacio-medina-veggiepizza-386394

Muñoz, P., Cacciotti, G., \& Cohen, B. (2018). The Double-Edged Sword of Purpose-Driven Behavior in Sustainable Venturing, Journal of Business Venturing, 33(2), 149-178. DOI: https://doi.org/10.1016/j.jbusvent.2017.12.005

Neergaard, H., \& Ulhøi, J.P. (Eds.). (2007). Handbook of Qualitative Research Methods in Entrepreneurship. Cheltenham \& Northampton: Edward Elgar Publishing Limited.

Nguyen, T.H.D., Chileshe, N., Rameezdeen, R., \& Wood, A. (2019). Stakeholder Influence Pathways in Construction Projects: Multicase Study. Journal of Construction Engineering \& Management, 145(9), 05019011-1- 05019011-14. DOI: https://doi.org/10.1061/(ASCE)CO.1943-7862.0001680

Partington, D. (Ed.). (2002). Essential Skills for Management Research. Londres: SAGE Publications.

Pierson, P. (2003). Big, Slow-Moving, and... Invisible. En J. Mahoney, \& D. Rueschemeyer (Eds.), Comparative Historical Analysis in the Social Sciences (pp. 177-207). Rhode Island: Cambridge University Press.

Quinton, S., \& Smallbone, T. (2006). Postgraduate Research in Business: A Critical Guide. Londres: SAGE Publications.

Rey-García, M., Calvo, N., \& Mato-Santiso, V. (2019). Collective Social Enterprises for Social Innovation. Understanding the Potential and Limitations of Cross-Sector Partnerships in the Field of Work Integration, Management Decision, 57(6), 1415-1440. DOI: https://doi.org/10.1108/MD-01-2017-0091

Sandberg, W., Robinson, R., \& Pearce, J. (2001). Why Small Businesses Need a Strategic Plan. Business \& Economic Review, 48(1), 12 - 15. Recuperado de: https://www.researchgate.net/publication/305002603_Why_small_businesses_need_a_strate gic_plan

Saunders, M., Lewis, P., \& Thornhill, A. (2009). Research Methods for Business Students. 5a edición. Essex: Prentice Hall. 
Vol. 9 | Investigación Cualitativa en Ciencias Sociales: Avances y Desafíos

Soutrisna, M., \& Goulding, J. (2019). Managing Information Flow and Design Processes to Reduce Design Risks in Offsite Construction Projects. Engineering, Construction and Architectural Management, 26(2), 267-284. DOI: https://doi.org/10.1108/ECAM-11-2017-0250

Stake, R. E. (1994). Case Studies. En N. Denzin, \& Y. S. Lincoln (Eds.), Handbook of Qualitative Research (pp. 236-247). Thousand Oaks, CA: Sage Publications.

Tesssem, B. (2018). What Causes Positive Customer Satisfaction in an Ineffectual Software Development Project. A Mechanism from a Process Tracing Case Study. International Journal of Information Systems and Project Management, 6(4), 83-98. DOI: https://doi.org/10.12821/ijispm060405

Tharenou, P., Donohue, R., \& Cooper, B. (2007). Management Research Methods. Camdbridge: Cambridge University Press.

Thietart, R.A. et al. (2001). Doing Management Research. A Comprehensive Guide. Londres: SAGE Publications.

Thomas, A. B. (2004). Research Skills for Management Studies. Londres: Routledge.

Timmer, S., \& Kaufmann, L. (2019). Do Managers' Dark Personality Traits Help Firms in Coping with Adverse Supply Chain Events? Journal of Supply Chain Management, 55(4), 67-97. DOI: https://doi.org/10.1111/jscm.12212

Van Evera, S. (1997). Guide to Methods for Students of Political Science. Ithaca: Cornell University Press.

Wang, C., Walker, E., \& Redmond, J. (2007). Explaining the Lack of Strategic Planning in SMEs: The Importance of Owner Motivation. International Journal of Organizational Behavior, 12(1), 1-16. Recuperado de: https://www.researchgate.net/publication/49277688

Weathington, B. L., Cunningham, C.J.L., \& Pittenger, D.J. (2012). Understanding Business Research. Somerset: John Wiley \& Sons.

Yin, R. K. (2008). Case Study Research: Design and Methods. Cuarta edición. Thousand Oaks, CA: Sage Publications. 